Cahiers de recherches médiévales

\title{
D'À rebours à Sainte Lydwine de Schiedam
}

Huysmans et les Primitifs d'une esthétique future

Jean-Pierre Guillerm

\section{(2) OpenEdition \\ Journals}

Édition électronique

URL : https://journals.openedition.org/crm/2493

DOI : $10.4000 / \mathrm{crm} .2493$

ISSN : 1955-2424

Éditeur

Honoré Champion

Édition imprimée

Date de publication : 12 décembre 1996

Pagination : 127-136

ISSN : 1272-9752

Référence électronique

Jean-Pierre Guillerm, «D'À rebours à Sainte Lydwine de Schiedam », Cahiers de recherches médiévales [En ligne], 2 | 1996, mis en ligne le 06 août 2008, consulté le 15 décembre 2022. URL : http:// journals.openedition.org/crm/2493; DOI : https://doi.org/10.4000/crm.2493 


\section{RM}

\section{D'A rebours à Sainte Lydwine de Schiedam, Huysmans et les Primitifs d'une esthétique future.}

Avant les plaisirs sulfureux du satanisme, Huysmans offre au lecteur de Là bas, ceux de la dissertation littéraire en trois points. Thèse, antithèse, synthèse, tout y est qui conduit à la nécessité d'un « naturalisme spiritualiste » qui seul pourra régénérer l'art modeme'

Ce manifeste pour une œuvre à venir ne s'étaie de nul exemple littéraire convenable. Dostoïevsky évoqué est aussi vite récusé pour trop de socialisme. Reste à changer de champ référentiel, à quitter l'écriture pour la peinture, le texte pour l'image constituée comme prophétique de ce qui aurait à advenir. Cette autorité conférée, non sans paradoxe, à la peinture, Huysmans la figure par la Crucifixion de Mathias Grünewald. ${ }^{2}$ De sorte qu'un Primitif de la peinture aurait à être le primitif d'une écriture dépassant et intégrant la contradiction actuelle du naturalisme sans âme et du spiritualisme sans corps.

La plénitude restituée de l'homme, tel que le christianisme le définit, s'est donc jadis figurée, incarnée dans une œuvre appartenant à ce corpus aux limites incertaines qui est celui des Primitifs ou encore des peintres du Moyen Age.

Découvrir chez les Primitifs médiévaux les exemples accomplis du «naturalisme spiritualiste » n'a rien d'une invention huysmansienne. Il y a belle lurette qu'un Ruskin a développé une telle interprétation et ceci dans une même perspective à la fois critique de la modemité et programmatique d'un avenir meilleur. Belle lurette que le Moyen Age pictural se trouve constitué comme repoussoir de l'académisme dérivant de la Renaissance, ceci n'étant qu'un aspect du grand dispositif réactionnaire qui oppose un Moyen Age mythisé à une modernité honnie. Cependant, rappeler Ruskin manifeste aussi le propre de Huysmans. En effet, comme Rio en France naguère, Ruskin ne pense foncièrement qu'à la peinture italienne. C'est à dire à des Primitifs qui, selon l'historiographie dominante, par leurs essais balbutiants auraient ouvert la voie au progrès vers le miracle de la Renaissance. Constituer en valeur absolue ces mêmes Primitifs bouleverse à coup sûr le parcours progressiste de la représentation esthétique établie mais maintient la légitimité picturale dans son champ exclusif : l'école italienne. Élire Grünewald, c'est s'aventurer sur les marges, qui plus est les marges allemandes, là où le Français entre Wagner et Bismarck hésite.

Choisir Grünewald comme autorité a la saveur d'une provocation. Loin des moyens âges italien et français, l'Allemand implique une sauvageriejusqu'alors irrécupérée par les discours canoniques ${ }^{3}$. C'est du fond noir de l'oubli et de la hantise que surgit la nouvelle

\footnotetext{
${ }^{1}$ Huysmans, J.K. Là-bas, Paris, Plon, 1908, p. 5.

${ }^{2}$ Le tableau passa rapidement du musée de Cassel, visité par Huysmans en 1888, à celui de Carlsruhe. Je ne peux tenir compte ici du texte publié en 1905 dans Trois primitifs, qui, à la suite du second voyage de Huysmans en Allemagne, décrit le polyptyque du musée de Colmar. Voir Joris-Karl Huysmans, Les Grünewald du musée de Colmar, édition critique par P. Brunel, A.Guyaux et C.Heck, Paris, Hermann, 1988.

${ }^{3}$ Cf. Huysmans, Les Grünewald du musée de Colmar, op. cit., p. 50 : « Quant à ses personnages, ils ont tous le type germain et il ne dérive pas davantage de l'art italien pour sa façon de déployer les éoffes. " D'autres s'étaient aventurés jusqu'à ces exotismes barbares, mais des Belges : Verhaeren, Jules Destrée.
} 
figure tutélaire, médiévale donc catholique, donc naturaliste mystique. Terme de la délibération, ultime marque de l'excès provocateur de la démarche et de la résistance à la future conversion:

Si je suis logique, j'aboutis au catholicisme du Moyen Age, au naturalisme mystique; ah non, par exemple, et si pourtant: ${ }^{4}$

L'abandon de l'école italienne, seule exemplaire, ouvre sur «cette Germanie du Moyen Age avec ses croyances sauvages, ses piétés barbares, ses coutumes mystiques ${ }^{5}$. Du moins demeure-t-on en chrétienté, voiredans l'intensité la plus forte, la plus «primitive» de cette chrétienté, une pureté première exempte de toute comption latino-bourgeoise. Un Moyen Age absolu en quelque sorte.

Dont il importe bien de dire qu'il n'a rien à voir avec une quelconque chronologie. L'espace sauvage ignore l'histoire et ses découpages. Huysmans sut fort bien que né vers 1450, Günewald mourut vers 1530, dates données dans Trois primitifs. Qu'il ait survécu à Raphaël n'empêche nullement Huysmans d'écrire alors de son peintre :

Le Laus perennis du Moyen Age revit en cet office incessant de la peinture que composa Grünewald. ${ }^{6}$

Grünewald est médiéval non historiquement mais essentiellement. Le Moyen Age des Primitifs est affaire d'âme et non de dates, affaire d'écart supposant l'âme. Cette apparente notion historique indexe moins un temps qu'un espace de marginalité psychique, sociale, culturelle, esthétique constitué en repoussoir d'une modernité fantasmée comme née de la Renaissance et prolongée sans rupture jusqu'au XIX siècle. Est médiéval tout ou presque qui est posé comme exempt du signe négatif de la Renaissance. Le primitif italien est d'emblée suspect d'être un « précurseur », d'où la fuite nécessaire, hors champ? .

Une dimension sociologique rudimentaire spécifie le Moyen Age huysmansien tout en contribuant à son extension indéfinie. Le Pauvre est médiéval et avec lui tous les vrais artistes, les isolés, les maudits, les exclus : Rops a une "âme de primitif à rebours ${ }^{8}$. Le Christ de Grünewald s'oppose au Christ de Saint Sulpice avatar du Christ de Raphaël :

le Christ des Riches, l'Adonis de Galilée, le bellâtre bien portant, le joli garçon aux mèches rousses, à la barbe divisée, aux traits chevalins et fades, que depuis quatre cents ans les fidèles adorent. $^{9}$

\footnotetext{
${ }^{4} L a ̈-b a s$, p. 11. 1870-1914, Lille, ANRT, 1982, pp. 542-579.

${ }^{6}$ Huysmans, Les Grünewald du musée de Colmar, p. 56. pp. 327-33.)

Huysmans, «Félicien Rops », Certains, 10 18, 1975, p. 343.

${ }^{9}$ Huysmans, Là bas, pp. 8-9.
}

${ }^{5}$ Verhaeren, "Les gothiques allemands ", L'Art modeme, 15 août 1886. Sur les sources du texte de Huysmans, voir C.Heck, "Grünewald et le culte des Primitifs septentrionaux chez Huysmans ", Huysmans Les Grünewald du musée de Colmar, pp.67-82, ainsi que pour un plus large contexte : J.P. Guillerm, Les peintures invisibles, L'héritage pictural et les textes en France et en Angleterre,

${ }^{7}$ En Bourgogne même, à la Chartreuse de Champmol, si le Moïse de Sluter vaut mieux que celui de Michel-Ange, " un colosse redondant et creux », les Prophètes annoncent trop la Renaissance : "gens qui se préoccupent plus de leurs propres affaires que de celles de Dieu. " (L'Oblat, Paris, Stock, 1903, 
Quatre cents ans exactement de domination d'une culture esthétique et religieuse de nantis, de bourgeois fades, à quoi s'oppose ce Christ des Pauvres. De sorte que la marginalité culturelle est aussi une marginalité sociale et que par elle, le Moyen Age puisse se prolonger jusqu'en plein XIX siècle comme revers noir d'une histoire des dominants.

L'irritant esthétisme religieux de Huysmans se fonde sur la fascination des exclusions qui déjà constituait l'univers expérimental de Des Esseintes. Une passion perverse pour la déterritorialisation estessentielle à l'acte esthétique huysmansien. Il faut être déserter le centre, s'ouvre alors l'espace médiéval. Ce centre, dans la peinture, Raphaël et la Renaissance italienne le présentent en passé toujours actif, toujours organisateur mythique des valeurs, toujours clef de l'idéologie culturelle. Le Moyen Age est un opérateur critique. Mythisé luimême, ilfigure le rejet de ce qu'implique le centre: l'équilibre, l'harmonie, le plaisir aussi. Le Moyen Age s'ouvre comme espace décentré d'errance et d'excès, de jouissance et de mort. Si le centre suppose le Beau, les marges imposent l'effroyable et l'adorable, l'horreur et l'extase. L'art huysmansien ne saurait être délectation, il est Passion, érotisme avant Bataille. Le Moi s'y perd entre sa décomposition et son exaltation.

C'est ce qu'écrit la longue séquence consacrée dans Là-basà la Crucifxion de Grünewald. Il importe très peu que cette description soit exacte par rapport au tableau ${ }^{10}$, d'ailleurs c'est moins le tableau qui est décrit que son apparition lorsque Durtal, les yeux clos, s'abîme dans le souvenir de sa vue à Cassel. Le descripteur est halluciné et cela convient au fantastique voulu du tableau: apparition de la Vie dans le visible de la mort, essence aporétique de l'art religieux médiéval. Mystère d'Incarnation.

Le texte est boursouflure métaphorique, excès d'écriture subvertissant le visible, le dégradant par l'étrange conjonction de la décomposition et de la nourriture: pectoraux beurrés par les sueurs, torse rayé de cercles de douves, chairs persillées de morsures de puces, lardées par les pointes de verges. Horreur de la Chair : le cadavre est festin, la crucifixion banquet abject. L'écriture tient moins à faire voir qu'à faire déraper le visible dans l'horreur nouant sacré et profane. De sorte que le visible en tant qu'il est sujet représenté, une crucifixion, ne soit que le générateur du carnaval fantasmatique de l'abjection. A ce jeu, la toile convoquée retournerait le lecteur de l'objet représenté vers le scripteur en rut, ivre en écriture de son naturalisme.

Il faut donc que cette peinture devienne chrétienne, qu'elle s'arrache au scripteur pour figurer, au delà, le mystère même du christianisme: la double nature du Christ, l'HommeDieu, qu'il soit sûr que «cette charogne éployée (est) celle d'un Dieu». Telle est bien la gageure de l'art religieux, reniée par une pratique vulgaire, païenne qui traite le problème par le Beau idéal et fait du Christ l'Adonis de Galilée. Alors qu'en revanche, Grünewald « réalise » le mystère:

Dans cette toile, se révélait le chef d'œuvre de l'art acculé, sommé de rendre l'invisible et le tangible, de manifester l'immondice du corps, de sublimer la détresse infinie de l'âme."

Si la métaphorisation descriptive a érigé l'écriture, une métaphorisation plus radicale s'instaure qui conjoint à l'évidence de la décomposition cadavérique, celle de la transfigura-

\footnotetext{
${ }^{10}$ Cf.C.Heck, art. cit., p. 73 : «Tant la place et la nature même des éléments que les caractères des personnages sont décrits avec exactitude, »

${ }^{11}$ Huysmans, Là bas, p. 10.
} 
tion glorieuse. Dans la tension la plus extrême entre le visible et l'invisible qui est son sens final.

Le Moyen Age est le lieu du symbolisme le plus radical, celui qui pose l'écart infini entre le signe et le sens. Ceci alors même et parce que le signe est aussi iconique que possible, saturant l'illusion représentative naturaliste. Or, avant même la conversion du scripteur/personnage, parce que les Primitifs sont essentiellement chrétiens, une lecture théologique convertit l'abjection en gloire et assigne au signifiant naturaliste un signifié mystique à la fois contradictoire et complémentaire dans la dramatique chrétienne du Salut. Coup d'état final du texte contre la peinture : la théologie du Salut par la Mort convertit figures et regard. Durtal voit ce qu'il ne voit pas :

à regarder ce Rédempteur de vadrouille, ce Dieu de morgue, cela changeait. De cette tête exulcérée filtrait des lueurs; une expression surhumaine illuminait l'effervescence des chairs, l'éclampsie des traits.

Avec et contre le visible de la peinture, dans la chambre noire des yeux clos, la figuration imagée du mystère se forme selon l'essence même du Moyen Age. Un change s'opère auquel aucun code ne concourt:

Cette charogne éployée était celle d'un Dieu et, sans auréole, sans nimbe, dans le simple accoutrement de cette couronne ébouriffée, semée de grains rouges par des points de sang, Jésus apparaissait, dans sa céleste superessence...

Apparition de l'étemel dans la misère du corps décomposé, sans signe autre que la misère corporelle, tout est dans le désir de foi qui suffit. Pas d'autre signe que celui, contradictoire, de l'horreur viscérale. Telle est la leçon du symbolisme médiéval: le sadisme comme figure de l'Infigurable. Le Moyen Age se constituant ainsi comme lieu du dépassement de la représentation mimétique que d'autres cherchent aussi, au même moment, dans le champ du Symbolisme littéraire et pictural. La Cathédrale, ne cite pas par hasard, après Hugues de Saint-Victor et saint Augustin, Mallarmé, même si Huysmans ajoute, avec humour, «rencontre pour le moins bizarre » ${ }^{12}$. L'enjeu esthétique de ce Moyen Age est bien de manifester que le sens n'est pas dans ce qu'on voit et reconnait mais au delà. Et que l'Invisible n'est que le signe nul de l'Indicible.

Certes, La Cathédraledonnerait à croire que la conversion n'a guère fait progresser Huysmans sur cette voie. Consacré à la symbolique médiévale, le texte, nouveau monstre littéraire, s'engorge d'un savoir encyclopédique. Excès cuistre, irritant et jubilatoire, d'exotismes nouveaux. Tout, de la cathédrale elle-même, son plan cruciforme et sa flèche semblent transposer la Crucifixion de Cassel, jusqu'au potager se met à signifier. Le Moyen Age est un livre, le Livre même, un monde où tout visible aurait été signe de l'Invisible. Rien ne peut être profane, là où la Foi ordonne le sens:

le Moyen Age qui savait que sur cette terre tout est signe, tout est figure, que le visible ne vaut que par ce qu'il recouvre d'invisible, le Moyen Age qui n'était pas, par conséquent, dupe

\footnotetext{
${ }^{12}$ Hurysmans, La Cathédrale, p. 85.
} 
comme nous le sommes, des apparences, étudia de très près cette science et dit d'elle la pourvoyeuse et la servante de la mystique. ${ }^{13}$

$\mathrm{Ni}$ auréole, ni nimbe, les symboles sont naturels, c'est toute la création, représentée au naturel, qui chante la gloire de Dieu. Ruskin n'avait rien dit d'autre et avec des excès qui ne sont pas moindres. A telle fleur, tel animal, telle pierrerie correspondent donc telle vertu ou tel vice. Tout visible est à lire pour qu'advienne au delà de son identification pratique, sa valeur morale, voire anagogique.

Les séances d'initiation à la symbolique médiévale de Durtal sont interrompues par un article que celui-ci est censé avoir rédigé à propos du Couronnement de la Vienge de Fra Angelico ${ }^{14}$.

Le collage de l'article dans la fiction narrative ramène donc vers l'Italie, aux bords d'abord exclus de la Renaissance. L'exception, on le verra, a sa raison. S'ensuit un exercice descriptif ouvert sur une nouvelle séquence encyclopédique déployant lasymboliquemédiévale des couleurs:

\section{Le blanc, symbole de l'Être supérieur, de la Vérité absolue (...) \\ Le bleu (...) rend la chasteté, l'innocence, la candeur (...) \\ Le rouge (...) traduit la charité, la souffrance et l'amour.... ${ }^{15}$}

Ces exercices mécaniques de "traduction" n'atteignent cependant que le parergonal, cette symbolique est "servante" du mystère central où s'abîment les codes. La mystique advient ici d'une perte radicale, d'uneinsignifiance. Ce qui est à convertir n'est plus la misère du corps mais celle de la représentation en ce centre traditionnel d'intérêt que constituent les visages:

Ce qui est moins explicable sur ce tableau, par exemple, c'est le choix limité des types de visage qu'il préféra; car ici, le symbole est inutile.

(Ses hommes) figurent sur ce tableau une placide colonie de très braves gens (...) (Ses fermmes) forment un cortège de types un peu gnangnan à cette Vierge au nez long, au crâne d'oiselle, agenouillée aux pieds du Christ. ${ }^{16}$

La déception touche à la fois la forme (la beauté) et l'expression. Le paradis n'a rien de la Beauté ni rien de l'horreur. Le terrain se trouve ainsi dégagé pour le coup de force interprétatif qui de cette déception fait le signe visible de la suréminente valeur mystique de l'œuvre:

Là, œ n'est plus un travail manuel, même souverain, ce n'est plus un ouvrage spirituel, vraiment religieux, ainsi que Roger Van der Weyden et Quentin Metsys en firent, c'est autre chose.

\footnotetext{
${ }^{13}$ Huysmans, La Cathédrale, p. 351.

${ }^{14}$ Au Louvre, peint pour un couvent de Fiesole ver 1434. Sur l'Angelico dans le contexte critique de la fin du siècle, cf. J.P. Guillerm, Les peintures invisibles, pp. 515-42.

${ }^{15}$ Huysmans, op. cit, pp. 132-3.

${ }^{16}$ Huysmans, op. cit, p. 134.
} 
Avec l'Angelico, un inconnu entre en scène, l'âme d'un mystique arrivé à la vie contemplative et l'effusant, ainsi qu'un pur miroir sur une toile (...)

Ses anges, ses saints, il les mène jusqu'à la vie unitive, jusqu'au suprême degré de la mystique (...) L'Angelico est le peintre de l'âme immergée en Dieu, le peintre de ses propres aîtres. ${ }^{17}$

Inutile là encore de se récrier, de consulter l'histoire et les dates. L'Angelico huysmansien n'est pas contemporain des premiers temps de la Renaissance, il est à peine Italien. Il est Moine. S'il est d'un temps, c'est de celui d'Angèle de Foligno, essentiellement médiéval et mystique.Le moine angélique se voit ainsi consacré comme le plus mystique des peintres, celui qui, parce que moine, s'est avancé le plus loin dans le «chemin de perfection ». Il peint la vie unitive et dépasse les laïcs Flamands et le laïc Grünewald qui apparaît, rétrospectivement, comme demeuré au premier stade mystique, celui de la vie purgative.

Au progrès de la peinture qui réglait l'historiographie commune se substitue le progrès mystique, redistribuant l'histoire, brouillant les écoles et les dates, établissant de nouvelles hiérarchies. Le Moine explique tout et d'abord l'annulation des signes mondains, l'indifférence sereine aux affects et aux corps. La banalité des figures se retoume de faiblesse en force, l'insignifiance en hyper-significance:

J'ai avancé que (ses saintes) avaient l'air insignifiant, et c'est vrai ; mais c'est que leurs traits, à elles aussi, se transforment et s'effacent sous l'épreinte divine! elles vivent noyées d'adoration, s'élancent, immobiles, vers le céleste Époux (...) Elle (la Vierge) est plus incomparable encore. Ici, les vocables de l'adulation défaillent, l'invisible apparaît sous les espèces des couleurs et des lignes (...) Elle est sans âge ; ce n'est pas une femme et ce n'est déjà plus une enfant. Et l'on ne sait même pas si Elle est adolescente, à peine nubile, une fillette, tant elle est sublimée, audessus de l'humanité, hors le monde, exquise de pureté, à jamais chaste! $!^{18}$

Vers l'invisible par l'insignifiance," ${ }^{19}$ c'est à dire par la perte des "vocables» et de leurs icônes. La représentation est creusée par un silence fondateur, de sorte que dans cet espace, tout signe codé est "péché ». Sainte Catherine d'Alexandrie, les "yeux pâmés », n'est «ni simple, ni candide ». Elle fait tache, fait revenir la femme dans la sainte, raphaélite déjà, non médiévale à coup sûr.

A moins qu'elle ne rappelle ces péchés du Moyen Age qui, au vrai, ne manquent pas. Là où la grâce surabonde... Huysmans poursuit d'une haine féroce les peintres de Cologne:

Alors, c'était ça, les Madones en fil de harpe, les vierges sublimées de Cologne! celle-là était bouffie, redondante, mafflue; elle avait un cou de génisse et des chairs de crème, en tôt-fait qui

\footnotetext{
${ }^{17}$ Huysmans, op. cit, p. 135.

${ }^{18}$ Huysmans, op. cit., p. 137-8. De même dans En route : « personne ne pourrait reconnaître en ces Trappistes des êtres prédestinés vivant hors la société modeme, en plein Moyen-Age, dans la fiance absolue d'un Dieu. S'ils ont des âmes qui ne ressemblent pas à celles des autres, ils ont, en somme, le visage et le corps des premiers venus.» (En route, Paris, Plon, 1961, p. 335). Croisant cette " insignifiance » avec la violence expressionniste, le porcher de la Trappe est, dans le mème texte, " un être angélique (...) il vit la vie unitive, l'âme ensevelie, noyée dans l'océan de la divine essence. " (En route, p. 279.)

${ }^{19}$ On est tout près de la « dissemblance " que G.Didi-Huberman analyse dans la peinture de l'Angelico et ses fondements théologiques, cf. G.Didi-Huberman, Fra Angelico Dissemblance et figuration, Paris, Flammarion, 1990.
} 
tremble quand on y touche (...) une cuvre méticuleuse et brillante, adroite, mais nullement religieuse ; il sentait la décadence, le travail fignolé, le compliqué et non le Primitif. ${ }^{20}$

Il faut donc discerner, ne pas succomber comme les autres au Dombild et à « l'apothéose du roublard et du bouffi ", chercher plus loin, en Franconie, en Souabe, les sauvages de génie, Grünewald, Zeitblom... ${ }^{21}$ Effet de snobisme, dégoût des femmes grasses, prurit antiallemand faisant retour, mais surtout marque claire que la conversion des signes relève bien du diktat critique. Est religieux, mystique, primitif, médiéval, ce que Huysmans déciare tel. Leçon inquiétante, à méditer, n'est «mystique» que ce que l'on proclame tel. La visée de l'indicible va de pair avec la tyrannie du dire critique.

Le Moyen Age pictural serait ainsi l'espace où la peinture s'imagine affranchie du culte humaniste de la « figure », de sa beauté, de son expression, pour trouver dans l'homme quelconque, dans le neutre figural, le support adéquat à l'épiphanie de l'Invisible. On oscille entre l'excès expressionniste de la misère charnelle et la neutralité sémiotique de la vie unitive, sans jamais s'arrêter au point juste de l'Homme qui serait celui de la Renaissance. L'horreur et la banalité appellent, symétriquement, le coup d'état dénégateur de l'interprète. Le Moyen Age huysmansien est l'espace constitué pour et par sa violence pulsionnelle, un champ ouvert pour la jouissance quasi terroriste de l'autorité du verbe.Ne pas ressusciter le passé, le créer selon son verbe : tâche d'écrivain, non d'historien, c'est clair. Et d'écrivain qui n'écrit que pour briser la loi de la représentation mimétique, sous l'effet de ce forcènement du sens imposé au visible.

Le discours sur la peinture médiévale renvoie d'ailleurs très exactement à celui que Huysmans peut tenir sur la littérature mystique comme aporie de l'écriture:

Nous en sommes réduits, pour qualifier ce mystérieux amour, à chercher nos comparaisons dans les acles humains, à infliger au Seigneur la honte de nos mots. Il nous faut recourir aux termes d'union, de mariage, de noces, à des vocables qui puent le suint! mais aussi comment énoncer l'inexprimable, comment, dans la bassesse d'une langue, désigner l'ineffable immersion d'une âme en Dieu. 22

Humilité nécessaire de l'écriture mystique : le désir de Dieu est «épreinte », il l'écrit sans vergogne. Humilité peut-être de toute écriture en tant qu'elle ne viserait que l'inexprimable. En contrepoint de cette bassesse, Hildegarde de Bingen indique un autre bord, l'hermétisme:

Son style apocalyptique a quelque chose de rétractile: il semble qu'il se recule et se referme davantage lorsqu'on veut l'ouvrir. ${ }^{23}$

La mystique ouvre sur l'inadéquation des signes linguistiques ou iconiques, sur l'« intraduisible». En conséquence, le déploiement encyclopédique des codes symboliques médiévaux est profusion omementale autour de l'irreprésentable central et fondateur du Sens. Le Moyen Age huysmansien est bien, à cet égard, le laboratoire d'une «poétique » modeme.

\footnotetext{
${ }^{20}$ Huysmans, La Cathédrale, p. 266-269.

${ }^{21}$ Encore que dans le polyptyque de Colmar, Huysmans ne supporte pas l'Annonciation...

${ }^{22}$ Huysmans, En route, p. 93.

${ }^{23}$ Huysmans, op. cit. p. 356.
} 
A l'Homme idéal de la Renaissance modemiste s'oppose l'Homme médiéval, le «Moine». Fra Angelico est l'homme neutralisé par l'infini de son essence en l'Autre. Cette « incamation" a son espace propre, soustrait au monde dans le monde même. Le Moyen Age est à la fois errance et clôture, deux antagoniques de l'homme urbain de la Renaissance. Le Moyen Age est le temps de l'homme retranché comme modèle d'accomplissement du destin terrestre. C'est à dire d'une vie pour la mort. La vocation médiévale est à la mort seule, anticipé en pleine vie recluse:

la seule fin qu'il était nécessaire, ici bas, d'atteindre, c'était d'entrer en relation directe avec le ciel et de devancer la morn. ${ }^{24}$ (Cathédrale, p.351)

C'est de cette anticipation dont témoigne l'art des Primitifs « vraie preuve du catholicisme»:

Alors, dans cet admirable Moyen Age, où l'art, allaité par l'Église, anticipa sur la mort, s'avança jusqu'au seuil de l'étemité, jusqu'à Dieu. le concept divin et la forme céleste furent devinés, entr' aperçus, pour la première et peut-être la dernière fois, par l'homme. ${ }^{25}$

Une mort annoncée dont l'expérience vive serait « bonheur» :

le bonheur consiste, certainement, à être interné dans un lieu très fermé, dans une prison bien close, où une chapelle est toujours ouverte. ${ }^{26}$

Tels sont les lieux et le temps de l'Art, écriture et peinture également mystiques qui ne peuvent être produites et reçues qu'au cloître. Ou dans ses approximations, car tel est l'horizon de conversion du retranchement esthète de Des Esseintes. Son musée imaginaire s'ouvrait déjà sur la cellule monastique, la chambre où :

il se figurait aisément alors qu'il vivait à cent lieues de Paris, loin du monde, dans le fin fond d'un cloître. ${ }^{27}$

L'expérience forcenée d'écriture que constitue $A$ Rebours programme les auto-fictions ultérieures constituées, livre après livre, comme conversion organisée de ses potentialités. La réclusion esthète de Des Esseintesprendra sens lorsqu'enfin Huysmans écrira la vie de sainte Lydwine de Schiedam. Non pas une moniale mais une laïque recluse dont la vie connue par quelques hagiographes obscurs se distribue en séquences disjointes où alternent l'horreur d'un corps vivant, sa décomposition et la naïveté de visions paradisiaques. La substitution, dans $A$ rebours, de la diégèse narrative à l'accumulation encyclopédique informe les écrits ultérieurs qui font de la compilation érudite le véritable moteur de l'écriture jusqu'à cette biographie compilée elle-même à partir de textes qui ne sont pas des «biographies» au sens où s'y déploierait le continu progressif d'une vie mais des kaléidoscopes a-chronologiques.

\footnotetext{
${ }^{24}$ Huysmans, op. cit, p. 351.

${ }^{25}$ Huysmans, op. cit, p. 8.

${ }^{26}$ Huysmans, op. cit, p. 284.

${ }^{27}$ Huysmans, A rebours, Paris, Charpentier-Fasquelle, 1918, p. 84.p. 89.
} 
Les vies écrites des mystiques médiévaux consignent moins l'histoire vécue que l'imprévisible des merveilles de Dieu, Angèle de Foligno et son «livre des visions» suffiraient à le manifester. La biographie se perd entre les excès altermés de la décomposition corporelle et de l'union en Dieu, entre la misère et la gloire. Les images fixes de la Crucifixion et du Couronnements'animent et se déploient en séquences difficilement agençables qui disent la mort vive de Lydwine. En une telle intrication que les figures rationalisées du progrès mystique s'y perdent:

Cette division des trois étapes de l'ascension mystique, si distincte chez les théologiens s'embrouille chez elle. Il n'est plus question de la halte du milieu, du relais illuminatif, mais des deux extrêmes, de la première et de la demière étape dans lesquelles elle semble, à une certaine époque, s'être également tenue. ${ }^{28}$

Le biographe à la fois organise un récit et subvertit cette forme par l'excès jouissif sadique des souffrances alternant avec les chromos pieux des visions paradisiaques. Une feinte näveté accueille les merveilles alternées de l'horreur et de la béatitude qui mettent en images l'anticipation de la mort. Les fleurs des saints se substituent à la botanique malade d'A rebours. Humble d'esprit, orgueilleux de style, l'écrivain néo-médiéval se soumet au folklore hagiographique pour ses écarts, sa différence par rapport au biographique humaniste. Le Moyen Age est encore ce qui permet de pénétrer la diégèse biographique de la puissance du symbole, de mettre à mort le récit bio-naturaliste pour y imposer les disjonctions de la mort. Entre l'excès expressionniste et le naïf de la vision paradisiaque, Sainte Lydwine de Schiedam recueille la leçon esthétique des Primitifs.

Cependant, le corpus huysmansien des Primitifs médiévaux ne cesse de communiquer avec un ensemble encore plus flou et totalement indénommé que nous appellerions «baroque ». Le musée imaginaire de Des Esseintes, là encore, remplit son office programmatique : gravures féroces de Van Luyken mais aussitoile du Greco qui, dans la chambrecellule, préfigure l'hallucination du Crucifié:

une ébauche désordonnée (...), un Christ aux teintes singulières, d'un dessin exagéré, d'une couleur féroce, d'une énergie détraquée(...)son Théocopuli (sic) dont l'atroce couleur rabrouait un peu le sourire de l'étoffe jaune et la rappelait à un ton plus grave....

La Cathédrale, sans hasard, insère dans son espace médiévaliste, la « remémoration » d'une autre œuvre baroque espagnole, le Saint François d'Assise peint par Zurbaran :

Le visage paraissait modelé, creusé dans la cendre et la bouche béait, livide, sous des yeux en extase, blancs, comme crevés. L'on se demandait comment ce cadavre qui n'avait que les os tenait debout et l'effroi venait, en songeant aux exorbitantes macérations, aux épouvantables pénitences qui avaient exténué ce corps et labouré les traits douloureux et ravis de cette face. ${ }^{30}$

Dans l'espace nocturne de la mémoire, le corps abîmé du mort-vif aux yeux d'aveugle érige l'évidence de la Gloire. C'est l'intime du rêveur qui à la fois suscite et éprouve la vérité

\footnotetext{
${ }^{28}$ Huysmans, Sainte Lydwine de Schiedam, Paris, Plon, 1901, p. 211.

${ }^{29}$ Huysmans, op. cit., p. 89

${ }^{30}$ Huysmans, La Cathédrale, p. 85. Il s'agit d'une toile du musée de Lyon, peinte vers 1645 , depuis les pillages napoléoniens et Théophile Gautier, Zurbaran était connu comme peintre mystique des couvents espagnols.
} 


\section{Jean-Pierre GUILLERM}

dans l'invisible de la toile vue. Or cet écart entre le visible et le sens renvoie en peinture à ce qui serait l'inadéquation radicale du langage à l'expérience unitive. De rares peintures baroques renvoient à de bien plus nombreuses citations d'écrits mystiques des $X V V^{e}$ et $X V I^{e}$ siècles mais ce corpus baroque, peintures et textes, n'est lui-même qu'un effet de déplacement de l'investigation médiévale. Le lieu médiéval huysmansien capte ce que nous désignerions comme baroque et qui est encore très largement indénommable au temps où écrit Huysmans.

La lutte engagée contre la trivialité naturaliste atteint en fait et non sans raison le Beau même par rapport à quoi le naturalisme instaure son scandale. Ce que Huysmans tend à défaire est l'immanence du sens à la représentation, telle serait la véritable bassesse de l'Art humaniste. Ce qui se construit dans les textes huysmansiens, que leur objet soit posé, souvent à tort, comme médiéval ou qu'il émerge de l'oubli méprisant de ce que nous appelons le baroque, c'est, indifféremment, la subversion de la coïncidence. Médiéval ou baroque, il importe que le texte puisse creuser le dit ou le vu d'un espace d'au delà qui est celui des épiphanies intimes de l'Invisible et de l'Indicible. Ce qui est donné dans l'expérience sensible doit appeler sa dénégation et ce n'est qu'un dérèglement de la représentation humaniste qui peut faire signe de l'au delà, Tout et Rien, Dieu dans le langage du croyant.

La réhabilitation esthétique des Primitifs n'est qu'un aspect de l'élargissement considérable du corpus pictural canonique qui caractérise le XIX ${ }^{e}$ siècle mais des Allemands du Moyen Age au Espagnols du Siècle d'or, ce qui marque les textes de Huysmans, c'est bien que cette extension s'intègre, à la fin du siècle, aux recherches symbolistes. La peinture et les textes mystiques sont des modèles d'interrogation de la représentation qui ouvrent sur les poétiques para-mystiques de notre temps. C'est Blanchot qui écrit que «l'écrivain est celui qui tient son pouvoir d'écrire d'une relation anticipée à la mort. ${ }^{31}$ Huysmans n'a rien dit d'autre de l'œuvre mystique. Mais en même temps, Huysmans ouvre sur les investigations amorcées du baroque. De Wölfflin à Severo Sarduy, le modèle de l'ellipse constitue un effet de corpus transsémiotique et largement diachronique où se cherche l'effondrement du cercle comme modèle de perfection. Une figure à deux pôles se substitue à l'autre si ancienne au pôle unique afin que le pôle visible ne puisse se concevoir sans un autre pôle obscur qu'on indentifiera comme lieu de révélation du Sens même.

L'errance médiévale dérive jusqu'au baroque historique et bien au delà, encore fallait-il passer par les marginalités médiévales pour mettre à mal la tyrannie naturaliste, au sens le plus large du terme. En cela, la Crucifixion de Grünewald, excédant la conscience critique de Huysmans lui-même, ouvre bien sur une poétique moderniste.

Jean-Pierre Guillerm

(Université Lille III)

${ }^{31}$ Maurice Blanchot, De Kafka à Kafka, Paris, Gallimard, Idées, 1981, p. 136. 